\title{
The Impact of Coherence Neurofeedback on Reading Delays in Learning Disabled Children: A Randomized Controlled Study
}

\author{
Robert Coben ${ }^{1 *}$,Emma Kate Wright ${ }^{2}$, Scott L. Decker ${ }^{2}$, and Tina Morgan ${ }^{3}$ \\ ${ }^{1}$ Integrated Neuroscience Services, Fayetteville, Arkansas, USA \\ ${ }^{2}$ University of South Carolina, Columbia, South Carolina, USA \\ ${ }^{3}$ NeuroRehabilitation and Neuropsychological Services, PC, Massapequa Park, New York, USA
}

\begin{abstract}
Introduction: Learning disabilities are a complex problem facing our society and educational system. Dyslexia, or reading disability, is one of the most common learning disabilities, impacting children and adults adversely in a myriad of ways. Traditional programs designed to teach reading enhancement are largely ineffective or require intensive therapy over long periods of time. Method: Forty-two school-aged participants were randomly assigned to experimental and control groups. The experimental group received qEEG-guided, individually tailored, twochannel coherence neurofeedback over the left hemisphere. This included two sessions per week for a total of 20 sessions. The control group received typical resource room instruction. All participants received pre- and post-educational measures focused on reading abilities. Results: Following the intervention period, the experimental group enhanced their reading scores, while the control group did not. Coherence neurofeedback led to an average enhancement of 1.2 grade levels in reading scores, but resource room instruction led to no such improvement at all. Conclusion: Coherence-based neurofeedback would appear to show promise and led to significant gains in reading that outpace those of traditional reading programs and most types of neurofeedback studied in the past. Future clinical and research work in this understudied area is recommended.
\end{abstract}

Keywords: Neurofeedback; coherence; reading; Dyslexia; learning disability

Citation: Coben, R., Wright, E. K., Decker, S. L., \& Morgan, T. (2015). The Impact of Coherence Neurofeedback on Reading Delays in Learning Disabled Children: A Randomized Controlled Study. NeuroRegulation, 2(4), 168-178. http://dx.doi.org/10.15540/nr.2.4.168

*Address correspondence to: Robert Coben, $\mathrm{PhD}$, Integrated Neuroscience Services, 92 West Sunbridge Drive, Fayetteville, AR 72703, USA. Email: drcoben@integrated-neuro.com

Copyright: (c) 2015. Coben et al. This is an Open Access article distributed under the terms of the Creative Commons Attribution License (CC-BY).

\section{Edited by:}

Rex Cannon, PhD, Neural Potential, Boynton Beach, Florida, USA

Reviewed by:

Rex Cannon, PhD, Neural Potential, Boynton Beach, Florida, USA Randall Lyle, PhD, Mount Mercy University, Cedar Rapids, lowa, USA

\section{Introduction}

Within the education system, learning disabilities are the most rapidly growing category for special education services, accounting for approximately $5 \%$ of the total student population in the United States (Cortiella \& Horowitz, 2014). Dyslexia, or specific reading disability, is one of the most common forms of learning disabilities, with prevalence rates ranging from $5 \%$ to $17 \%$ (Shaywitz, 1998; Shaywitz, Fletcher, Shaywitz, 1994). Dyslexia involves impairment in reading for both children and adults that would not be anticipated when considering an individual's cognitive ability, motivation, or education (Shaywitz, 1998). Individuals who struggle with reading fall on a continuum, ranging from minimal to severe skill deficits. According to the National Reading Panel (2000), the skills necessary for learning to read include phonemic awareness (understanding sounds or phonemes that make up a spoken word), phonics (associating sounds or phonemes to letters of language, then using this understanding to form words), reading fluency (quickly and accurately reading words), and reading comprehension (ability to read text and process it for its meaning). Additionally, individuals with Dyslexia struggle with spelling, vocabulary, and written expression (Cortiella \& Horowitz, 2014).

Further, research suggests that there are neurobiological underpinnings of specific cognitive deficits that are associated with learning disabilities 
(Eden \& Zeffiro, 1998; Ramus, 2004; Shaywitz \& Shaywitz, 2004). McCandliss and Noble (2003), provided a thorough review of the neurological deficits associated with developmental Dyslexia. Based on the understanding that phonological processing is a fundamental procedure required for reading, many researchers studied the superior temporal gyrus (STG) due to its association with this process. McCandliss and Noble outlined 11 studies examining the STG and its functioning with comparisons between individuals with and without Dyslexia. Studies have found that individuals with Dyslexia show less activity within the STG when challenged by phonological processing. Further, another brain region associated with developmental Dyslexia, that was highlighted in the literature according to McCandliss and Noble, is the left occipito-temporal extrastriate visual system. This visual system is located by the fusiform gyrus, which is responsible for the automaticity of word recognition in skilled readers. McCandliss and Noble discussed several functional neuroimaging studies that indicated the left fusiform gyrus' specific association with visual word form perception, and its lack of involvement with perception of other visual stimuli. Additionally, it was reported that the visual word form area was not associated with false graphemes or auditory words, but only "visually presented alphabetic characters," supporting the specific function of the left fusiform gyrus (McCandliss \& Noble, 2003, p. 200).

Temple et al. (2003) conducted a study that supported the findings reviewed by McCandliss and Noble (2003). This study conducted functional Magnetic Resonance Imaging (fMRI) scans on 20 children with Dyslexia before and after completing an intervention focused on improving auditory processing and oral language skills. Results found differences in the left temporo-parietal cortex and the left inferior frontal gyrus when performing phonological tasks; where after the intervention, children with Dyslexia had improved reading and increased activity in the mentioned brain areas with task performance.

These studies outlined above by McCandliss and Noble (2003) and Temple et al. (2003) illustrate the neurobiological basis for reading difficulty. Identification of brain regions associated with Dyslexia is pivotal for developing intervention methods that specifically target neurobiological functioning as a means to improve reading ability. Dyslexia's high occurrence within adult and child populations draws attention to the need for research to focus on efficacious interventions methods for reading difficulties.

\section{Interventions and Efficacy}

The Orton-Gilligham (OG) approach (Sheffield, 1991) is a common reading intervention method that allows trained teachers, tutors, or specialists to provide a structured, multisensory, and cumulative method to individuals in need of reading instruction. The multisensory element of this technique incorporates auditory, kinesthetic, and visual pathways for learning to help children use phonemic awareness, the alphabetic principles (sound to symbol relationship), decoding, encoding, fluency, and comprehension (Ritchey \& Goeke, 2006). These researchers reviewed OG and OG-based programs for reading instruction and found mixed results after reviewing 12 studies. A total of five of the 12 studies indicated that when compared to control interventions, OG instruction resulted in enhanced reading outcomes, while one indicated no significant differences after including covariates, two reported that alternate instruction was more beneficial, and four reported that $O G$ instruction was beneficial for at least one reading skill, but not all (Ritchey \& Goeke, 2006). Overall, Ritchey and Goeke were unable to clarify the effectiveness of OG or OG-based intervention strategies. Further, the What Works Clearinghouse (WWC) reached the same conclusion as Ritchey and Goeke. WWC examines the efficacy of research centered on education. OG studies reviewed by WWC did not meet WWC evidence standards, indicating that the efficacy of OG-based interventions is inconclusive at this time (U.S. Department of Education, Institute of Education Sciences [IES], 2010a).

The Wilson Reading System (WRS; Wilson, 1998) is a remedial program for both children and adults who have had difficulty with other teaching techniques for learning how to read. The WRS uses OG principles to teach phonemic awareness, alphabetic principles, vocabulary, fluency, and comprehension with multisensory principles. The WRS program was designed to be implemented over the course of one to three years through a cumulative 12-step sequence (Wilson \& O'Connor, 1995). In a data review completed by Wood (2002), large cohort study reports using the WRS program were analyzed and found to have an average improvement of 0.38 standard deviations $(S D)$ on the Woodcock Reading Mastery Test total reading cluster over a 1-year intervention period. Improvements were seen across all subtests on the test, including Word Identification, Word Attack, Passage Comprehension, and the Basic Skills 
Cluster. The WWC reviewed nine studies that implemented the WRS program, and reported that WRS may have a positive impact on an individual's understanding of alphabetic principles, but little to no effect on fluency and comprehension skills (U.S. Department of Education, Institute of Education Sciences [IES], 2010b). The WRS program appears to be minimally efficacious, but does not consistently improve all skill areas necessary for higher-level reading.

Lindamood-Bell Learning Processes (LMB; 2005) were designed to focus on building reading and comprehension skills through helping individuals understand the "process" of language. This goal is achieved through helping learners acquire proficiency in phonemic awareness, symbol imagery (phonologic and orthographic processing), and concept imagery (oral and written language). According to the clinical statistics from LMB, word reading improved by $0.60 \quad S D$ and sentence/paragraph reading improved by $0.35 S D$ over the course of one year of instruction. The WWC reviewed one research study for the Lindamood Phonemic Sequencing (LiPS) program, and found that LiPS may have a positive impact on an individual's understanding of alphabetic principles and reading fluency, no effect on reading comprehension, and possible negative effects on writing skills (U.S. Department of Education, Institute of Education Sciences [IES], 2008).

Another important intervention method to consider with Dyslexia is reading instruction within the context of a special education resource classroom, as opposed to a general education classroom. In a longitudinal study conducted by Bentum and Aaron (2003), reading achievement of children with learning disabilities who were instructed in a resource room was examined. The first group of children with learning disabilities contained 230 individuals who had a previous diagnosis of a reading disability and were re-evaluated after 3 years of resource room instruction. The second group of children with learning disabilities contained a total of 64 children who were in a resource room for a total of 6 years, where re-evaluation occurred twice, each after 3 years. Pre- and post-test scores for reading achievement and IQ testing were compared for both groups. Results found that instruction within the learning disability resource room did not improve word recognition or reading comprehension skills. Additionally, students within the resource room actually experienced a decrease in spelling scores for both student groups. Finally, results indicated that children who had received special education in the resource room for 6 years demonstrated a decrease in performance on verbal IQ testing (Bentum \& Aaron, 2003). Bentum and Aaron's findings are alarming in that the goal of the special education resource room is to provide a more individualized, intensive education setting to help improve difficulties in learning. If special education resource rooms are ineffective, then what other intervention methods can we look to for helping struggling readers? One new and revolutionary intervention method that has been studied in the literature for learning disabilities involves the use of Neurofeedback (NF).

\section{EEG and Neurofeedback with Dyslexia}

Electroencephalography (EEG) has become a widely accepted and utilized brain imaging method, but the scope of how EEG can be used for research has yet to be established (Michel \& Murray, 2012). EEG records the electrical activity of the brain with electrodes on the scalp and has recently been used in studies of individuals with learning disabilities. Learning disability studies using EEG have seen clear differences in brain activity when comparing individuals with and without learning disabilities. One example is that decreased connectivity is found between brain regions responsible for visual symbols and language sounds for individuals with Dyslexia (Shaywitz, Gruen, \& Shaywitz, 2007), likely illustrating why it is often harder for struggling readers to apply alphabetic principles. Additionally, EEG research has found that children with learning disabilities, specifically Dyslexia, have increased theta activity and decreased alpha and beta activity when compared to children without learning disabilities (Fernández et al., 2002; Lubar et al., 1985). With this knowledge researchers have started to use EEG biofeedback or NF to help treat learning disabilities. NF trains participants to direct their own EEG activity through operant conditioning.

Fernández et al. (2003) conducted a study to examine how NF treatment with children who have learning disabilities would affect their EEG activity and behavior. The study included 10 children with learning disabilities that had theta to alpha absolute power ratios that were higher than average. Then, to maintain similar IQ, socioeconomic status, gender, and the Test of Variables of Attention (TOVA) scores, subjects were divided into a control and experimental group. The TOVA is a computerized assessment measure that helps screen for attention disorders like AttentionDeficit/Hyperactivity Disorder (ADHD). NF was provided to both groups for a total of twenty 30-min sessions, with approximately two sessions per week. 
The control group received noncontingent reinforcement, while the experimental group had NF that targeted a subject's brain region with the highest theta/alpha ratio. Reinforcement in the experimental group was contingent on the theta/alpha ratio falling below a threshold, which when achieved would signal a sound for reinforcement. Results found improved performance on IQ testing, as well as decreased delta, theta, alpha, and beta bands' absolute power in the experimental group. These changes were not found with the control group, signifying the possible effectiveness of $\mathrm{NF}$ as a treatment option for children with learning disabilities.

In a study conducted by Orlando and Rivera (2004), NF was administered to sixth-, seventh-, and eighthgrade students identified with learning disabilities. Pre- and post-test cognitive and reading measures were administered to determine if NF would improve basic reading, reading comprehension, total reading, and IQ scores. Students were randomly assigned to either the control or experimental group, with a total of 10 students in the experimental condition and 14 in the control condition. Students in the experimental group received, on average, no more than $45 \mathrm{~min}$ of NF per session, for a total of 28 sessions. Results found that NF had a positive outcome on the treatment condition with an increase of $0.36 S D$, versus students within the control condition who had a decrease of $0.33 S D$. The NF administration protocol was not specified within the study.
Additionally, Thornton (2006) has reported that auditory and reading memory increased by more than three $S D$ when using a targeted protocol based on qEEG for children with learning disabilities and/or ADHD. With research supporting the efficacy of NF as a treatment method for learning disabilities, the current research study seeks to examine how EEG coherence training might impact reading abilities in students diagnosed with Dyslexia.

\section{Method}

\section{Participant Characteristics}

This study involved 42 children who were previously, and independently, identified with a learning disability (Dyslexia). The Winthrop University Hospital IRB approved participation in this study and the procedures used. All subjects' parents or legal guardians provided written consent. Participants were interviewed and completed neuropsychological and educational testing by a qualified administrator. To be diagnosed with a learning disability, educational age equivalent scores had to be 1.5 to 2 years delayed compared to chronological age. None of the participants had any other neurological or psychiatric diagnosis that would account for the delays. All 42 participants had delays on measures of reading or reading comprehension. The demographic characteristics of the samples are provided in Table 1.

\section{Table 1}

Demographics for the total sample, broken down by experimental (coherence) and control (resource) groups.

\begin{tabular}{|c|c|c|c|c|c|c|c|c|c|}
\hline \multicolumn{10}{|c|}{ Descriptives } \\
\hline & & \multirow[b]{2}{*}{$N$} & \multirow[b]{2}{*}{ Mean } & \multirow[b]{2}{*}{$S D$} & \multirow[b]{2}{*}{ Std. Error } & \multicolumn{2}{|c|}{ 95\% Confidence Interval for Mean } & \multirow[b]{2}{*}{ Minimum } & \multirow[b]{2}{*}{ Maximum } \\
\hline & & & & & & Lower Bound & Upper Bound & & \\
\hline \multirow{2}{*}{ Age } & Resource & 21 & 11.954 & 1.7669 & .3856 & 11.150 & 12.759 & 8.8 & 15.1 \\
\hline & Total & 42 & 12.030 & 1.8065 & .2787 & 11.467 & 12.593 & 8.8 & 15.5 \\
\hline Handedness & Coherence & 21 & 1.190 & 0.4020 & .0880 & 1.010 & 1.370 & 1.0 & 2.0 \\
\hline \multirow[t]{3}{*}{ Gender } & Coherence & 21 & 1.190 & 0.4020 & .0880 & 1.010 & 1.370 & 1.0 & 2.0 \\
\hline & Resource & 21 & 1.240 & 0.4360 & .0950 & 1.040 & 1.440 & 1.0 & 2.0 \\
\hline & Total & 42 & 1.210 & 0.4150 & .0640 & 1.080 & 1.340 & 1.0 & 2.0 \\
\hline \multirow[t]{2}{*}{ Race } & Coherence & 21 & 1.100 & 0.3010 & .0660 & 0.960 & 1.230 & 1.0 & 2.0 \\
\hline & Resource & 21 & 1.140 & 0.3590 & .0780 & 0.980 & 1.310 & 1.0 & 2.0 \\
\hline
\end{tabular}




\section{Instruments}

All participants were administered a battery of neuropsychological and intellectual tests. Reading abilities were measured with the Woodcock-Johnson Tests of Achievement III (WJ III; Woodcock, McGrew, \& Mather, 2007) and the Gray Oral Reading Test-4 (GORT-4; Wiederholt \& Bryant, 2001). The WJ III reading scores included letter word identification, reading fluency, and reading comprehension subtests. Letter word identification asks participants to read a list of words to measure word identification. Letter word identification has a reliability of .91 for children and adolescents and a reliability of .94 for adults. Reading fluency asks participants to read statements and determine whether the statement is true or false. Three minutes are given to complete as many questions as possible. Questions gradually increase in difficulty level. Reading fluency has a reliability of .90 for children, adolescents, and adults. Passage comprehension begins with matching a pictograph representation of a word with the actual picture of the object followed by multiple choice. Multiple choice format requires a person to point to a picture. The final section of the test requests participants to identify a word missing from a paragraph. Passage comprehension has a reliability of .83 for children and adolescents and .88 for adults. The GORT- 4 is a reading test measuring fluency and comprehension. A person is asked to read a paragraph out loud and then answer questions about the paragraph. GORT-4 is reported to have .90 reliability. The tests were administered to control and experimental group participants before and after treatment.

All subjects also underwent qEEG assessment data collection before and after intervention. EEG data was obtained under two conditions, eyes closed and eyes open. A stretchable electrode cap embedded with 19 sensors attached to the scalp was used to collect data, with frontal reference, prefrontal ground, and linked ears. Each recording lasted 20 min, where 10 min were spent in both conditions. EEG acquisition involved recording and digitizing EEG readings based on the International 10/20 System of electrode placement utilizing the Deymed Diagnostic (2004) TruScan 32 Acquisition EEG System. This system included 32 channels with sampling at 128 cycles per second and filtering between $0.1-40 \mathrm{~Hz}$. All recordings were done with impedance less than $5 \mathrm{k} \Omega$. The common mode rejection ratio for this system is $102 \mathrm{~dB}$ and the isolation mode rejection ratio is $140 \mathrm{~dB}$. The reliability and validity of quantitative EEG (qEEG) have been sufficiently assessed and confirmed
(Thatcher, 2010). We have previously shown qEEG data to be useful in impacting outcome of NF intervention for children on the autistic spectrum (Coben \& Myers, 2010; Coben \& Padolsky, 2007). All EEG data was subjected to manual artifacting procedures conducted by a professional with more than 30 years' experience. qEEG data analysis included the use of the Neurometric Analysis System (NxLink, 2001; John, Prichep, Fridman, \& Easton, 1988) and NeuroGuide (Applied Neuroscience, Inc.; Thatcher, Walker, Biver, North, \& Curtin, 2003), both of which are FDA approved. Specific coherence analyses were conducted with the NeuroRep (Hudspeth, 1999) connectivity analysis system.

The experimental group participated in NF sessions as the active treatment. The NeuroCybernetics EEGer Training System (NeuroCybernetics Inc., 2006) was used to perform connectivity-guided EEG biofeedback training. The sensors (Grass Silver Disc 48" Electrodes with SafeLead protected terminals; Grass SafeLead, 2006) were applied to the subject's scalp to measure EEG activity. The signal was then fed back to the subject in visual and aural form based on relative amplitude/threshold values. The visual feedback consisted of simple graphics (presented in the form of computer games), providing a continuous display of the ratio of amplitude to threshold for each stream of data. The aural reward consisted of a prerecorded sound file of a short 0.25 -s beep, occurring no more often than once per every half second and activating when specific amplitude/coherence conditions were met (NeuroCybernetics Inc., 2006).

\section{Procedure}

All 42 patients diagnosed with a learning disability underwent neuropsychological, IQ, and educational testing, and qEEG assessment prior to intervention in a private clinical practice setting. Educational testing and qEEG assessment were completed following intervention. Reading scores for the two groups were based on the WJ III and the GORT-4. All subjects were already receiving resource room assistance at school but no other active interventions. The 42 participants were randomly assigned to an experimental and control group with 21 in each group. The experimental group received $\mathrm{NF}$ training as the active treatment and the control group received their resource room assistance but no NF.

Neurofeedback Protocols

The 21 participants that made up the experimental group received 20 sessions of two-channel 
connectivity-guided EEG coherence NF two times a week. Treatment was personalized to each individual on the basis of his or her original qEEG findings for power and coherence. Based on each participant's qEEG analysis, areas showing the most prominent hypocoherence (low coherence) were targeted for training. All maximal hypocoherence connections indicated by the qEEG were located on the left side of the brain. Of the 21 participants receiving coherence training, the most common connection found to be too low was the occipitalparietal region to the frontal-temporal. The second most common hypocoherence identified was the parietal to medial temporal connections. Less common was the hypocoherence connection between temporal-parietal to frontal regions with only three of the subjects receiving this training. All coherence protocols also included inhibiting excessive amplitude based on significant qEEG findings over those regions.

Protocol designs were different for each individual. Rewarding for coherence increases was matched to the frequencies showing the greatest hypocoherences. For most participants this included delta, theta, and often alpha bands as well. This was combined with amplitude inhibits for low frequencies (delta-theta) for 17 of 21 subjects. There was also a second inhibit for most subjects that included alpha and low beta frequencies. Lastly, a third inhibit was common for higher frequencies, often in the $20-30 \mathrm{~Hz}$ range. These individual sessions were run for no longer than 20 minutes of actual training.

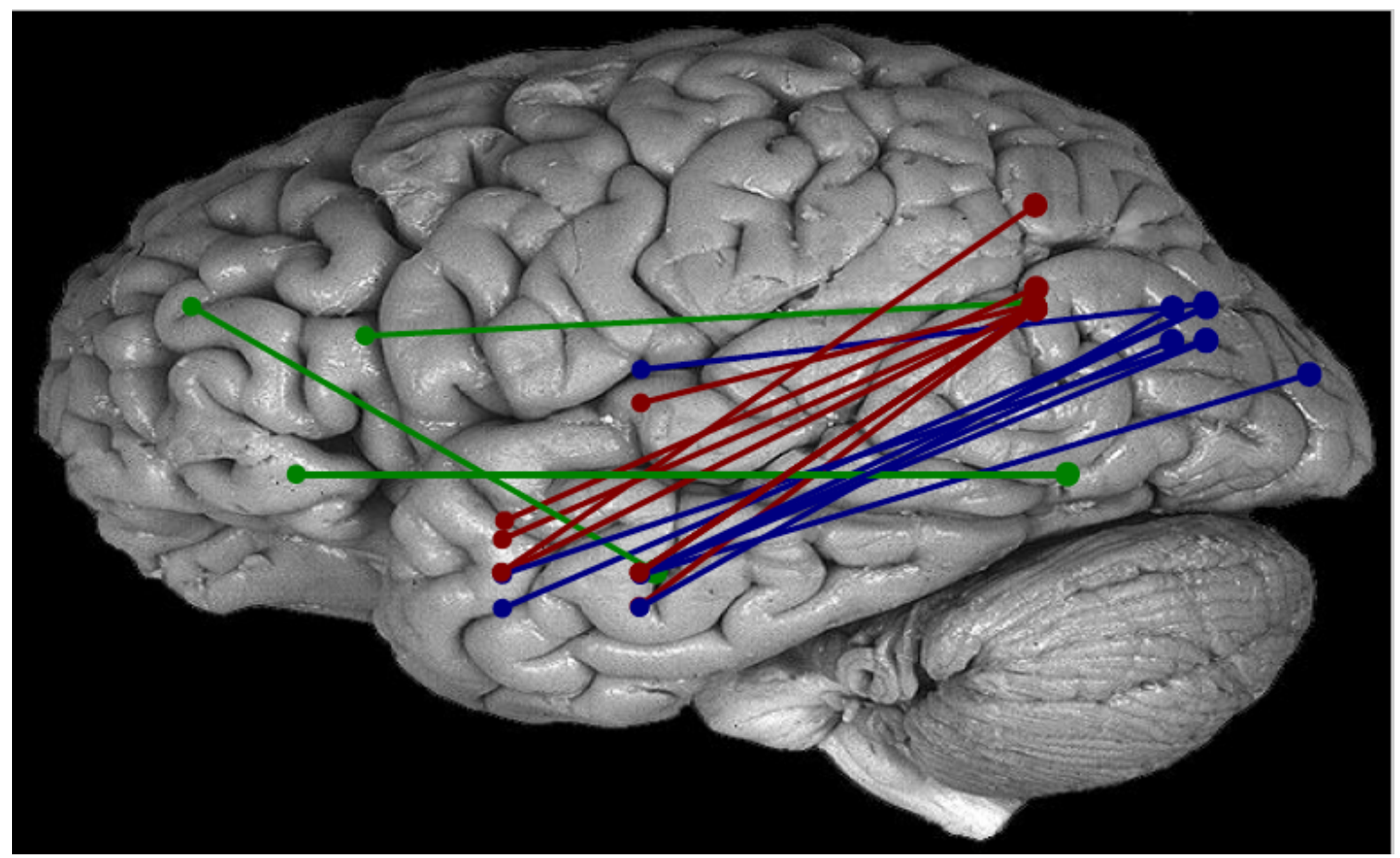

Figure 1. Graphic representation of two-channels involved in NF protocol for each subject in the experimental group. Represented are those trained from occipital-temporal (blue), parietal-temporal (red), and temporal-parietal-frontal (green).

\section{Results}

The primary aim of this research was to compare if reading scores changed significantly in the experimental group as compared to the control group following the intervention period. When one compares these two groups, there was no significant difference for age of the samples $(t=0.27$, ns), handedness $(z=0.38, \mathrm{~ns})$, gender $(z=0.38, \mathrm{~ns})$, race $(z=0.48$, ns), medication $(t=0$, ns), or baseline reading scores $(t=0.99$, ns). When baseline scores in reading were compared to the subject's age (reading delay), there was a mild indication that the control group $(2.7 \pm 0.28)$ had less of a delay than the experimental group $(3.22 \pm 0.52)$ but this difference was not statistically significant $(t=$ $1.85, p=.07$ ). As a result, one may conclude that any differences in reading scores and change in 
reading scores are unlikely to be due to these factors. Detailed demographic descriptive statistics are shown in Table 1 above and descriptive statistics for delays in reading are shown in Table 2 below.

Table 2

Reading delay in years for the total sample, experimental (coherence) and control (resource) groups.

\begin{tabular}{|c|c|c|c|c|c|c|c|c|c|}
\hline \multicolumn{10}{|c|}{ Descriptives } \\
\hline & & \multirow[b]{2}{*}{$N$} & \multirow[b]{2}{*}{ Mean } & \multirow[b]{2}{*}{$S D$} & \multirow[b]{2}{*}{ Std. Error } & \multicolumn{2}{|c|}{$95 \%$ Confidence Interval for Mean } & \multirow[b]{2}{*}{ Minimum } & \multirow[b]{2}{*}{ Maximum } \\
\hline & & & & & & Lower Bound & Upper Bound & & \\
\hline \multirow{2}{*}{ Reading Delay } & Resource & 21 & 2.697 & 0.6073 & .1325 & 2.421 & 2.974 & 1.9 & 4.1 \\
\hline & Total & 42 & 2.958 & 0.9414 & .1453 & 2.665 & 3.252 & 1.6 & 5.3 \\
\hline
\end{tabular}

An analysis of variance (ANOVA) was performed with group (coherence vs. resource) as the independent variable and pre- and post-treatment reading scores serving as the dependent variables. As presented above, there was no significant difference between reading scores at baseline, but there was at post-tests after the intervention period $(F=7.557, p<.01)$. Post-hoc tests showed that this change in reading scores was due to the fact that the experimental group improved upon their reading scores, while the control group did not.

\section{Table 3}

Descriptive statistics for reading age equivalent (reading age 1) at baseline and at follow-up (reading age 2).

\begin{tabular}{|c|c|c|c|c|c|c|c|c|c|}
\hline \multicolumn{10}{|c|}{ Descriptives } \\
\hline & & \multirow[b]{2}{*}{$N$} & \multirow[b]{2}{*}{ Mean } & \multirow[b]{2}{*}{$S D$} & \multirow[b]{2}{*}{ Std. Error } & \multicolumn{2}{|c|}{ 95\% Confidence Interval for Mean } & \multirow[b]{2}{*}{ Minimum } & \multirow[b]{2}{*}{ Maximum } \\
\hline & & & & & & Lower Bound & Upper Bound & & \\
\hline \multirow{2}{*}{ Reading Age 1} & Resource & 21 & 9.257 & 1.4045 & .3065 & 8.618 & 9.896 & 6.6 & 12.0 \\
\hline & Total & 42 & 9.071 & 1.2192 & .1881 & 8.691 & 9.451 & 6.6 & 12.0 \\
\hline Reading Age 2 & Coherence & 21 & 10.129 & 1.2021 & .2623 & 9.581 & 10.676 & 8.0 & 12.0 \\
\hline
\end{tabular}

\section{Table 4}

One-way ANOVA (group x reading scores).

\begin{tabular}{|c|c|c|c|c|c|c|}
\hline \multicolumn{7}{|c|}{ ANOVA } \\
\hline & & $\begin{array}{l}\text { Sum of } \\
\text { Squares }\end{array}$ & df & Mean Square & $F$ & Sig. \\
\hline \multirow[t]{2}{*}{ Reading Age 1} & Between Groups & 1.449 & 1 & 1.449 & 0.974 & .330 \\
\hline & Total & 60.946 & 41 & & & \\
\hline Reading Age 2 & Between Groups & 11.947 & 1 & 11.947 & 7.557 & .009 \\
\hline
\end{tabular}

Comparison of the groups showed a significant difference in reading change scores (time 1 - time 2). When age equivalent scores on reading tests were used as the dependent variable there was a significant difference between the two groups ( $t=$
$12.8, p<.001$ ). The experimental group (coherence training) enhanced their reading scores by a mean of 1.243 years, while the control group had a small decrease in reading scores of 0.2 years. These changes are depicted in Figure 2. 


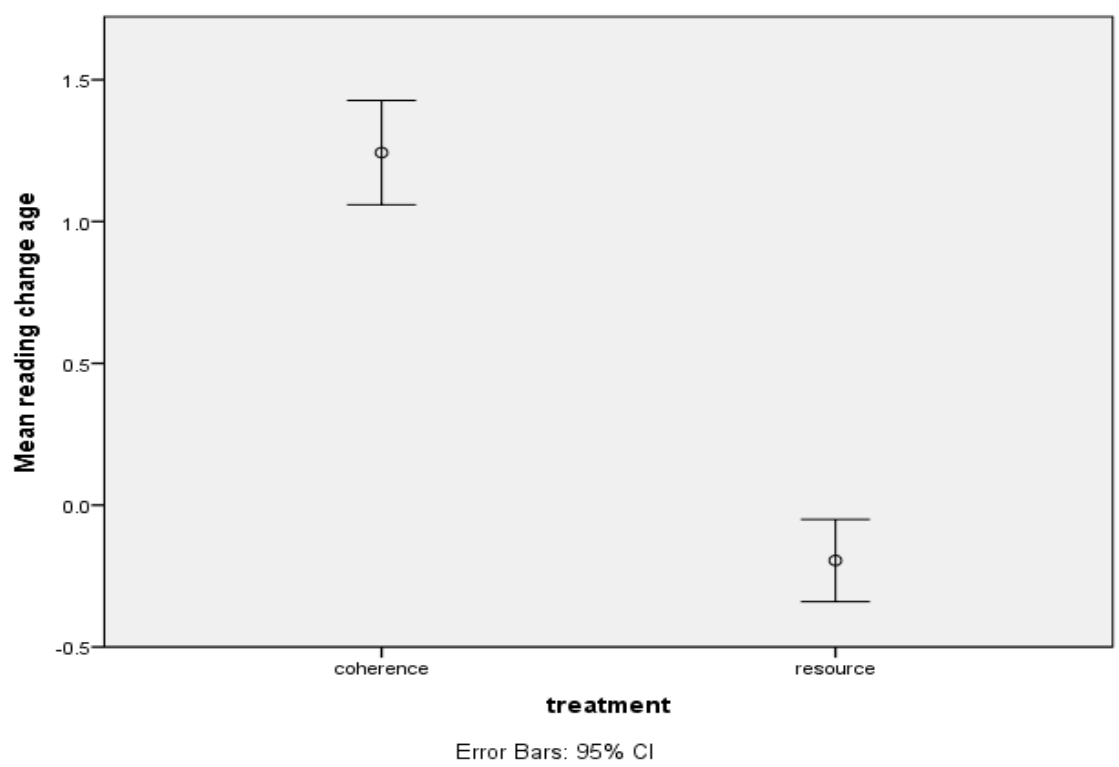

Figure 2. Change in age equivalent reading scores by group.

We also calculated an index of delay in reading called percentage reading delay based on their age and how far delayed they are in reading scores. We then measured their improvement on this index over the course of training/intervention. Not surprisingly, there was again a difference between the groups $(t=$
13.45, $p<.001$ ) with the experimental group showing greater change. The experimental group (coherence training) improved by $40 \%$, while the control group (resource room) declined by $7 \%$. These results are shown in Figure 3.

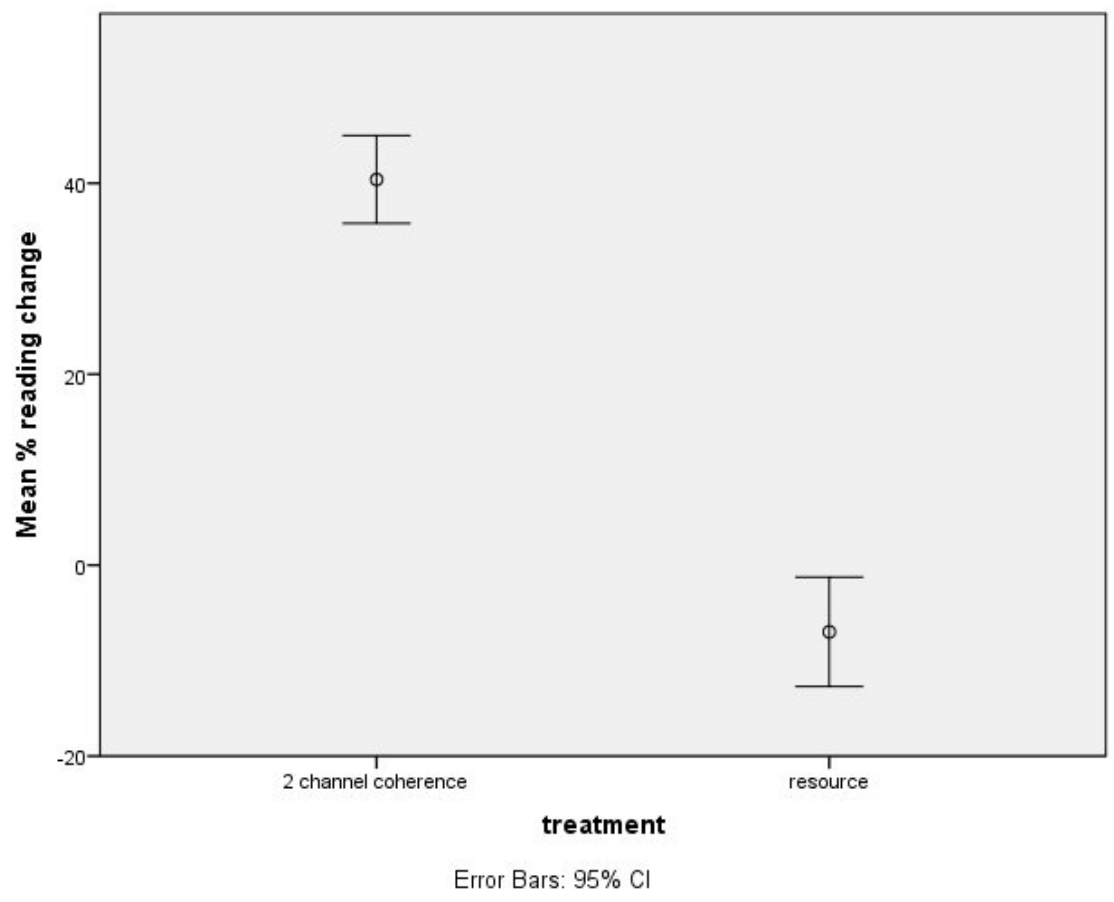

Figure 3: Change in percentage reading delay by group. 


\section{Conclusion}

Remedial approaches for children with learning disabilities have been found to have limited effectiveness. This is important given the number of children in need of remedial services and the escalating cost to treat children with learning disabilities in schools. As such, it is of tremendous importance to not only identify effective interventions but also interventions that are financially sustainable.

The current study used an experimental design to examine the effectiveness of NF on improving reading performance in children identified with reading disorders in comparison to a matched control group receiving standard educational interventions. Forty-two children with reading delays were randomly assigned to an experimental group that received coherence-based NF and a control group that received resource room instruction. Those that received NF improved upon their reading scores, while those in the control group did not. The average improvement of the experimental group was 1.2 grade levels in reading scores over the course of only $20 \mathrm{NF}$ sessions (total treatment duration of only 10 hours).

These findings compare favorably and exceed the efficacy of other interventions available to help children with reading problems. Traditional reading programs like the OG approach have not been able to show consistent efficacy (Ritchey \& Goeke, 2006; U.S. Department of Education, Institute of Education Sciences [IES], 2010a). Similarly, the Fast ForWord language intervention program has not proven to have efficacy for reading improvement compared to comparison groups (Strong, Torgerson, Torgerson, \& Hulme, 2011). The Lindamood-Bell learning program has been shown to be mildly beneficial, but requires at least a full year of instruction to achieve these aims (Lindamood-Bell Learning Processes, 2005). It is also very interesting to note that interventions like resource room instruction tend to have very little impact on reading abilities in children with reading delays (Vaughn \& Wanzek, 2014). Our findings are certainly consistent with this.

With this in mind, the search for effective reading interventions is critical. There is some evidence that NF training can enhance academic abilities in children with learning disabilities. Orlando and Rivera (2004) showed a moderate effect from NF compared to a no treatment control group, but even this effect took an average of seven months or longer. Nazari, Masonezhad, Hashemi, and Jahan
(2012) showed in six single subject cases of NF changes in coherence associated with improved academic abilities. Of course, their paper lacked experimental design to draw any firm conclusions. Lastly, Breteler, Arns, Peters, Giepmans, and Verhoeven (2010) conducted a study of 19 children using qEEG-guided NF training but found no impact on reading abilities, but improvements in spelling only. Their potential impact may have been mitigated by pairwise instead of multivariate coherence analyses and its impact on the type of training they chose (see Coben, MohammedRezazadeh, \& Cannon, 2014). Our current study has double the sample size of these others and has corrected many methodological flaws discussed above. Our findings suggest a dramatic enhancement of reading abilities in a short time frame and suggest an important avenue for future clinical and research endeavors.

Despite these encouraging findings, our research has some of its own limitations that should be addressed with future work. Our sample may have been biased by the hope of clinical gain, even though they knew there was only a $50 \%$ chance of receiving the active treatment. While our randomization to experimental and control groups was a positive feature, all participants knew which group they were in and there was never an attempt to blind them or the experimenters. Future work that is single- or double-blinded in some way may overcome this weakness. Lastly, this data was gathered at a time when we did two-channel coherence training. Since this time, other advanced techniques have been developed in the field of NF including multivariate four-channel NF (Coben, 2014), low-resolution brain electromagnetic tomography (LORETA) NF (Cannon, Congedo, Lubar, \& Hutchens, 2009) and others that may be able to enhance the efficacy of this work even further.

\section{References}

Bentum, K. W., \& Aaron, P. G. (2003). Does reading instruction in learning disability resource rooms real work?: A longitudinal study. Reading Psychology, 24, 361-382. http://dx.doi.org/10.1080/02702710390227387

Breteler, M. H. M., Arns, M., Peters, S., Giepmans, I., \& Verhoeven, L. (2010). Improvements in spelling after QEEGbased neurofeedback in Dyslexia: A randomized controlled treatment study. Applied Psychophysiology and Biofeedback, 35(1), 5-11. http://dx.doi.org/10.1007/s10484-009-9105-2

Cannon, R., Congedo, M., Lubar, J., \& Hutchens, T. (2009). Differentiating a network of executive attention: Loreta Neurofeedback in anterior cingulate and dorsolateral prefrontal cortices. International Journal of Neuroscience, 119(3), 404-441. http://dx.doi.org/10.1080 100207450802480325 
Coben, R. (2014, October). Four Channel Multivariate Coherence Training: Rationale and Findings. [Plenary session]. Presented at the ISNR 22nd Annual Conference, San Diego, CA.

Coben, R., Mohammed-Rezazadeh, I., \& Cannon, R. L. (2014). Using quantitative and analytic EEG methods in the understanding of connectivity in autism spectrum disorders: A theory of mixed over- and under-connectivity. Frontiers in Human Neuroscience, 8, 45. http://dx.doi.org/10.3389 /fnhum.2014.00045

Coben, R., \& Myers, T. E. (2010). The relative efficacy of connectivity guided and symptom based EEG biofeedback for autistic disorders. Applied Psychophysiology and Biofeedback, 35(1), 13-23. http://dx.doi.org/10.1007/s10484009-9102-5

Coben, R., \& Padolsky, I. (2007). Assessment-Guided Neurofeedback for Autistic Spectrum Disorder. Journal of Neurotherapy, 11(1), 5-23. http://dx.doi.org/10.1300 IJ184v11n01_02

Cortiella, C., \& Horowitz, S. H. (2014). The State of Learning Disabilities: Facts, Trends, and Emerging Issues (3rd ed.). New York: National Center for Learning Disabilities.

Deymed Diagnostic (2004). TruScan EEG Specifications. Retrieved from http://www.deymed.com/products-aservices/truscan-eeg/specifications

Eden, G. F., \& Zeffiro, T. A. (1998). Neural systems affected in developmental dyslexia revealed by functional neuroimaging. Neuron, 21(2), 279-282. http://dx.doi.org/10.1016/S08966273(00)80537-1

Fernández, T., Herrera, W., Harmony, T., Díaz-Comas, L., Santiago, E., Sánchez, L., ... Valdés, R. (2003). EEG and behavioral changes following neurofeedback treatment in learning disabled children. Clinical EEG and Neuroscience, 34(3), 145-152. $\quad$ http://dx.doi.org/10.1177 $/ 155005940303400308$

Grass SafeLead (2006). Genuine Grass precious metal recording electrodes: SafeLead. Retrieved from http://www.grasstechnologies.com

Hudspeth, W. J. (1999). NeuroRep QEEG analysis and report system. Los Osos, CA: Neuropsychometrix.

John, E. R., Prichep, L. S., Fridman, J., \& Easton, P. (1988). Neurometrics: Computer-assisted differential diagnosis of brain dysfunctions. Science, 239, 162-169.

Lindamood-Bell Learning Processes. (2005). Lindamood-Bell Learning Processes 2005 clinical statistics. Retrieved from http://www.lindamoodbell.com/downloads/pdf/research/clinica I\%20stats\%202005.pdf

Lubar, J. F., Bianchini, K. J., Calhoun, W. H., Lambert, E. W., Brody, Z. H., \& Shabsin, H. S. (1985). Spectral analysis of EEG differences between children with and without learning disabilities. Journal of Learning Disabilities, 18(7), 403-408. http://dx.doi.org/10.1177/002221948501800708

McCandliss, B. D., \& Noble, K. G. (2003). The development of reading impairment: a cognitive neuroscience model. Mental Retardation and Developmental Disabilities Research Reviews, 9(3), 196-205. $\quad$ http://dx.doi.org/10.1002 /mrdd.10080

Michel, C. M., \& Murray, M. M. (2012). Towards the utilization of EEG as a brain imaging tool. Neurolmage, 61(2), 371-385. http://dx.doi.org/10.1016/j.neuroimage.2011.12.039

National Reading Panel, National Institute of Child Health and Human Development. (2000). National Reading Panel: Teaching children to read: An evidence-based assessment of the scientific research literature on reading and its implications for reading instruction (NIH Publication No. 004754). Retrieved from https://www.nichd.nih.gov/publications /pubs/nrp/Documents/report.pdf

Nazari, M. A., Masonezhad, E., Hashemi, T., \& Jahan, A. (2012). The effectiveness of neurofeedback training on EEG coherence and neuropsychological functions in children with reading disability. Clinical EEG and Neuroscience, 43(4), 315-322. http://dx.doi.org/10.1177/1550059412451880

NeuroCybernetics Inc. (2006). Specific EEGer technical parameters. Canoga Park, CA: NeuroCybernetics Inc.

NxLink (2001). Neurometric analysis system. Richland, WA: NxLink Ltd.

Orlando, P. C., \& Rivera, R. O. (2004). Neurofeedback for elementary students with identified learning problems. Journal of Neurotherapy, 8(2), 5-19.

Ramus, F. (2004). Neurobiology of dyslexia: A reinterpretation of the data. Trends in Neurosciences, 27(12), 720-726. http://dx.doi.org/10.1016/j.tins.2004.10.004

Ritchey, K. D., \& Goeke, J. L. (2006). Orton-Gillingham and Orton-Gilliangham-based reading instruction: A review of the literature. The Journal of Special Education, 40(3), 171-183. http://dx.doi.org/10.1177/00224669060400030501

Shaywitz, S. E. (1998). Dyslexia. The New England Journal of Medicine, 338(5), 307-312. http://dx.doi.org/10.1056/NEJM199801293380507

Shaywitz, S. E., Fletcher, J. M., \& Shaywitz, B. A. (1994). Issues in the definition and classification of attention deficit disorder. Topics in Language Disorders, 14(4), 1-25. http://dx.doi.org /10.1097/00011363-199408000-00003

Shaywitz, S. E., Gruen, J. R., \& Shaywitz, B. A. (2007). Management of dyslexia, its rationale, and underlying neurobiology. Pediatric Clinics of North America, 54(3), 609623. http://dx.doi.org/10.1016/j.pcl.2007.02.013

Shaywitz, S. E., \& Shaywitz, B. A. (2004). Reading disability and the brain. Educational Leadership, 61(6), 6-11.

Sheffield, B. B. (1991). The structured flexibility of OrtonGillingham. Annals of Dyslexia, 41(1), 41-54. http://dx.doi.org/10.1007/BF02648077

Strong, G. K., Torgerson, C. J., Torgerson, D., \& Hulme, C. (2011). A systematic meta-analytic review of evidence for the effectiveness of the 'Fast ForWord' language intervention program. Journal of Child Psychology and Psychiatry, 52(3), 224-235. http://dx.doi.org/10.1111/j.1469-7610.2010.02329.x

Temple, E., Deutsch, G. K., Poldrack, R. A., Miller, S. L., Tallal, P., Merzenich, M. M., \& Gabrieli, J. D. E. (2003). Neural deficits in children with dyslexia ameliorated by behavioral remediation: Evidence from functional MRI. Proceedings of the National Academy of Sciences of the United States of America, 100(5), 2860-2865. http://dx.doi.org/10.1073 /pnas.0030098100

Thatcher, R. W. (2010). Validity and Reliability of Quantitative Electroencephalography. Journal of Neurotherapy, 14(2), 122-152. http://dx.doi.org/10.1080/10874201003773500

Thatcher, R. W., Walker, R. A., Biver, C. J., North, D. N., \& Curtin, R. (2003). Quantitative EEG Normative Databases: Validation and Clinical Correlation. Journal of Neurotherapy, 7(3-4), 87121. http://dx.doi.org/10.1300/J184v07n03_05

Thornton, K. (2006). Subtype analysis of learning disability by quantitative electroencephalography patterns. Biofeedback, 34(3), 106-113

U.S. Department of Education, Institute of Education Sciences (IES). (2008). Lindamood Phonemic Sequencing (LiPS). Retrieved from http://ies.ed.gov/ncee/wwc/pdf /intervention_reports/wwc_lindamood_121608.pdf

U.S. Department of Education, Institute of Education Sciences (IES). (2010a). Orton-Gillingham-Based Strategies (Unbranded). Retrieved from http://ies.ed.gov/ncee /wwc/pdf/intervention reports/wwc ortongill 070110.pdf

U.S. Department of Education, Institute of Education Sciences (IES). (2010b). Wilson Reading System. Retrieved from http://ies.ed.gov/ncee/wwc/pdf/intervention_reports/wwc_wils on_070110.pdf

Vaughn, S., \& Wanzek, J. (2014). Intensive interventions in reading for students with reading disabilities: Meaningful impacts. Learning Disabilities Research and Practice, 29(2), 46-53. http://dx.doi.org/10.1111/ldrp.12031 
Wiederholt, J. L., \& Bryant, B. R. (2001). GORT 4 Gray Oral Reading Tests Examiner's Manual. Austin, TX: Pro-Ed.

Wilson, B. A. (1998). Matching student needs to instruction: Teaching reading and spelling using the Wilson Reading System. In S. A. Vogel \& S. M. Reder (Eds.), Learning disabilities, literacy, and adult education (pp. 213-235). Baltimore, MD: P. H. Brookes Publishing Co.

Wilson, B. A., \& O'Connor, J. R. (1995). Effectiveness of the Wilson Reading System used in public school training. In C. Mclntyre and J. Pickering (Eds.), Clinical Studies of Multisensory Structured Language Education (pp. 247-254). Salem, OR: International Multisensory Structured Language Education Council.
Wood, F. (2002). Wilson literacy solutions: Evidence of effectiveness. (Unpublished data compilation report). Oxford, MA: Wilson Language Training Corp.

Woodcock, R. W., McGrew, K. S., \& Mather, N. (2007). Woodcock-Johnson III Tests of Achievement. Rolling Meadows, IL: Riverside Publishing.

Received: November 17, 2015

Accepted: November 20, 2015

Published: December 9, 2015 